\title{
ON THE STRUCTURE OF THE CANONICAL MODULE OF THE REES ALGEBRA AND THE ASSOCIATED GRADED RING OF AN IDEAL
}

\author{
S. ZARZUEI.A
}

Dedicated to the memory of Pere Menal

Abstract

In this note we give the description of a morphism related with the structure of the canonocal module of the Rees algebra $R(I)$ of an ideal $I$ in a local ring. $\Lambda$ s an application we obtain Ikeda's criteria for the Gorenstcinness of $R(I)$ and a result of HerzogSimis-Vasconcelos characterizing when the canonical module of $R(I)$ las the expected form.

Let $(A, \mathrm{~m})$ be a Noetherian local ring and $I$ be an ideal of $A$. In [4] Ikeda characterized the Gorensteinness of the Rees algebra $R(I)=$ $\rightarrow I^{n} t^{n}$ by means of the canonical module of $A$ and the canonical module $n \geq 0$

of the associated graded ring $g r_{A}(I)=\oplus I^{n} / I^{n+1}$, under the assumptions that $R(I)$ is Cohen-Macaulay and $\operatorname{grade}(I) \geq 2$. If in particular $A$ is Cohen-Macaulay this characterization means that if $h l(I) \geq 2$, the Rees algebra $R(I)$ is Gorenstein if and only if the ground ring $A$ is Gorenstein and the associated graded ring $g^{r} A(I)$ is Gorenstein with $a$-invariant $a\left(g r_{A}(I)\right)=-2$.

On the other hand in [3| Herzog-Simis-Vasconcelos investigated the canonical modules of $R(I)$ and $g^{r} A(I)$, where $I$ is an ideal in a local Cohen-Macaulay ring $(A, m)$. They were specially interested in characterizing when the canonical module of the Rees algebra $R(I)$ is isomorphic to the $R(I)$-submodule of the polynomial ring $A[t]$ generated by $1, t, \ldots, t^{n n}$, where $m \geq 0$, or to the ideal $I R(I)$. Then it is said that the canonical module of $R(I)$ has the expected form, that occurs if in particular $R(I)$ is Gorenstein. 
These results have been recently used to study the Gorensteinness of the Ress algebras and associated graded rings of powers of ideals, see [2].

Our deal in this paper is to give a common point of view for both results. For this, and mainly inspired in [3], we first give the description of a morphism of graded $R(I)$-modules

$$
\Gamma: \underset{n \geq 2}{\oplus}\left(K_{R(I)}\right)_{n} \rightarrow K_{R(n)}
$$

where $K_{R(l)}$ is the canonical module of the Recs algebra $R(I)$. By means of this morphism $\Gamma$ some information about the structure of $K_{R(I)}$ can be transfered to the canonical module of the associated graded ring $g T_{A}(I)$, and conversely. This is done is section 1, proposition (1.1). Then, as a main application, we obtain in section 2 the above mentioned results of Ikeda and Herzog-Simis-Vasconcelos.

The existence of a morphism with similar properties as $\Gamma$ for multigraded Rees algebras has been obtained by H. Hiri (Helsinki).

\section{The main result}

We shall use the book [1] as a reference for unexplained results and terminology. Let $R=\underset{n \geq 0}{\oplus} R_{n}$ be a Noetherian graded ring defined over a local ring $R_{0}$. If $L$ is a finitely generated graded $R$-module then the Krull dimension of $L, \operatorname{dim}(L)$, satisfies

$$
\operatorname{dim}(L)=\sup \left\{i \mid \underline{H}_{N}^{i}(L) \neq 0\right\},
$$

where $\underline{H}_{N}^{i}(L)$ are the $i-t h$ graded local cohomology modules of $L$ with respect to $N$, the maximal homogeneous ideal of $R$. The a-invariant of $L$ is then defined by

$$
a(L):=\sup \left\{n \mid\left(\underline{H}_{N}^{\operatorname{dim}(L)}(L)\right)_{n} \neq 0\right\} .
$$

Since $\underline{H}_{N}^{\operatorname{dim}(L)}(L)$ is an artinian graded $R$-module, $a(L)$ is a well defined integer.

Assume that $R$ has a canonical module, $K_{R}$. Passing to the completion if necessary and by local duality one has that

$$
a(R)=-\inf \left\{n_{1} \mid\left(K_{R}\right)_{n} \neq 0\right\},
$$

and $R$ is Gorenstein if and only if $R$ is Cohen-Macaulay and $K_{R} \simeq$ $R(a(R))$. 
From now on $(A, \mathrm{~m})$ will be a local ring and $I$ an ideal of $A$. We shall use the following notations:

$S=R(I)=\underset{n \geq 0}{\oplus} I^{n} t^{n} \subset A[t]$, the Rees algebra of $I$;

$S_{+}=\underset{n \geq 1}{\oplus} I^{n} t^{n n}=t(I R(I))=(t I) R(I)$.

$M=\mathrm{m} \oplus S_{+}$, the maximal homogeneous ideal of $R(I)$,

$G=g r_{A}(I)=\underset{n \geq 0}{\oplus} I^{n} / I^{n+I}$, the associated graded ring of $I$,

$K_{S}=K_{R(I)}, K_{G}=K_{g r_{A}(I)}, K_{A}$ the canonical modules of $R(I)$, $g r_{A}(I)$ and $A$.

Since $g r_{A}(I)=R(I) / I R(I)$ and $A=R(I) / S_{+}$, the canonical modules $K_{g F_{A}(I)}$ and $K_{A}$ exist if the canonical module $K_{R(I)}$ exists. Assume moreover that $R(I)$ is Cohen-Macaulay and $h(l)>0$. By $[4,(2.1)]$ the $\alpha$-invariant $a\left(g r_{A}(I)\right)$ is negative, and from the same proof it can be deduced that $a(R(I))=-1$.

Proposition (1.1). Let $(A, \mathrm{~m})$ be a local ring and $I \subset A$ be an ideal of $A$ with ht $(I)>0$. Assume that the Rees algebra $R(I)$ is Cohen-Macaulay and has a canonical module $K_{R(I)}$. Put

$$
\left(K_{R(I)}\right)_{+}:=\underset{r \geq 2}{\bigoplus}\left(K_{R(I)}\right)_{n}
$$

Then there exists a morphism of graded $R(I)$-modules

$$
\Gamma:\left(K_{R(I)}\right)_{+} \rightarrow K_{R(I)}
$$

such that:

(i) $\Gamma$ is injective of degree -1 .

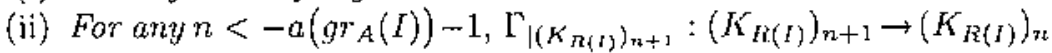
is bijective.

(iii) For any element $\beta \in\left(K_{R(I)}\right)_{+}, l^{\prime}(\beta)$ is the only element in $K_{R(I)}$ such that $s \beta=(t s) \Gamma(\beta)$ for ary $s \in I R(I)$.

(iv) For any $r \geq 1$ and any $\alpha \in K_{R(l)} . \Gamma\left(\left(t^{r} y\right) \alpha\right)=\left(t^{r-1} y\right) \alpha$ for any $y \in I^{r}$.

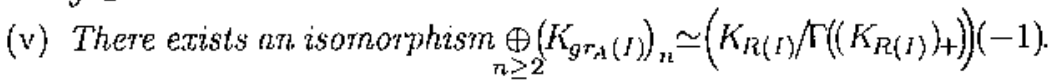

Proof: Consider the exact sequences of graded $S$-modules

$$
\begin{aligned}
& 0 \rightarrow S_{+} \rightarrow S \rightarrow A \rightarrow 0 \\
& 0 \rightarrow I S \rightarrow S \rightarrow G \rightarrow 0 .
\end{aligned}
$$


Dualizing with $K_{S}$ we get the exact sequences

$$
\begin{aligned}
& 0 \rightarrow \underline{\operatorname{Hom}}_{S}\left(A, K_{S}\right) \rightarrow \underline{\operatorname{Hom}}_{S}\left(S, K_{S}\right) \rightarrow \underline{\operatorname{Hom}}_{S}\left(S_{+}, K_{S}\right) \rightarrow \\
& \underline{\operatorname{Ext}}_{S}^{1}\left(A, K_{S}\right) \rightarrow \operatorname{Ext}_{S}^{1}\left(S, K_{S}\right)=0
\end{aligned}
$$

and

$$
\begin{aligned}
0 \rightarrow \underline{\operatorname{Hom}_{S}}\left(G, K_{S}\right) \rightarrow & \underline{\operatorname{Hom}}_{S}\left(S, K_{S}\right) \rightarrow \underline{\operatorname{Hom}}_{S}\left(I S, K_{S}\right) \rightarrow \\
& \underline{\operatorname{Ext}}_{S}^{1}\left(G, K_{S}^{\prime}\right) \rightarrow \underline{\operatorname{Ext}}_{S}^{1}\left(S, K_{S}\right)=0 .
\end{aligned}
$$

By $[1,(36.8)] K_{S}$ is a Cohen-Macaulay $S$-module with $\operatorname{depth}\left(K_{S}\right)=$ $\operatorname{dim}(S)=\operatorname{dim}(A)+1$, hence given that $\operatorname{dim}(A)=\operatorname{din}(G)$ we obtain $\underline{\operatorname{Hom}}_{S}\left(A, K_{S}\right)=\operatorname{Hom}_{S}\left(G, K_{S}\right)=0$. On the other hand by [1, (36.14)] $K_{A} \simeq \operatorname{Ext}_{S}^{1}\left(A, K_{S}\right), K_{G} \simeq \operatorname{Ext}_{S}^{1}\left(G, K_{S}\right)$, thus we have exact sequences

$$
\begin{aligned}
& 0 \rightarrow \underline{\operatorname{Hom}}_{S}\left(S, K_{S}\right) \stackrel{\pi}{\rightarrow} \underline{\operatorname{Hom}}_{S}\left(S_{+}, K_{S}\right) \stackrel{\psi}{\rightarrow} \underline{K}_{A} \rightarrow 0 \\
& 0 \rightarrow \underline{\operatorname{Hom}}_{S}\left(S, K_{S}\right) \stackrel{\sigma}{\rightarrow} \underline{\operatorname{Hom}}_{S}\left(I S, K_{S}\right) \stackrel{\varphi}{\rightarrow} \underline{K}_{G} \rightarrow 0
\end{aligned}
$$

where $\pi$ and $\sigma$ are the obvious restriction maps.

Furthermore $\underline{\text { Hom }}_{S}\left(S, K_{S}\right)$ may be identified with $K_{S}$ by the map that in degree $n$ is given by

$$
\begin{aligned}
\operatorname{Hom}_{S}\left(S, K_{S}\right)_{n} & \rightarrow\left(K_{S}\right)_{n}, \\
f & \rightarrow f(1)
\end{aligned}
$$

thus we may finally writo exact sequences

$$
\begin{aligned}
& 0 \rightarrow W_{S} \stackrel{\pi}{\rightarrow} \underline{\operatorname{Hom}}_{S}\left(S_{+}, K_{S}\right) \stackrel{\psi}{\rightarrow} K_{A} \rightarrow 0 \\
& 0 \rightarrow K_{S} \stackrel{\sigma}{\rightarrow} \underline{\operatorname{Hom}}_{S}\left(I S, K_{S}\right) \stackrel{\varphi}{\rightarrow} K_{G} \rightarrow 0 \text {. }
\end{aligned}
$$

Consider the morphism of graded $S$-modules

$$
\begin{aligned}
\tau: \underline{\operatorname{Hom}}_{S}\left(S_{+}, K_{S}\right) & \rightarrow \underline{\operatorname{Horn}}_{S}\left(I S, K_{S}\right) \\
f & \rightarrow \tau(f): I S \rightarrow K_{S} \\
s & \rightarrow \tau(f)(s)=f(t s) .
\end{aligned}
$$

Since $S_{+}=t(I S), \tau$ is an isomorphism of degree 1 and for any $n$ there exists a diagram with exact sequences

$$
\begin{gathered}
0 \rightarrow\left(K_{S}\right)_{n} \stackrel{\pi}{\rightarrow} \underline{\operatorname{Hom}}_{S}\left(S_{+}, K_{S}\right)_{n} \stackrel{\psi}{\rightarrow}\left(K_{G}\right)_{n} \rightarrow 0 \\
\tau \downarrow \\
0 \rightarrow\left(K_{S}\right)_{n+1} \stackrel{\sigma}{\rightarrow} \underline{\operatorname{Hom}}_{S}\left(I S, K_{S}\right)_{n+1} \stackrel{\varphi}{\rightarrow}\left(K_{A}\right)_{n+1} \rightarrow 0 .
\end{gathered}
$$


Define $\omega:=\tau \pi . \quad K_{A}$ is a graded $S$-module reduced to degree 0 while $\left(K_{S}\right)_{n}=0$ for any $n \leq 0$, hence

$$
\pi: K_{S} \rightarrow \underset{n \geq 1}{\oplus} \underline{\operatorname{Hom}}_{S}\left(S_{+}, K_{S}\right)_{n}
$$

is an isomorphism and

$$
\omega: K_{S} \rightarrow \underset{n \geq 2}{\oplus} \underline{\operatorname{Hom}}_{S}\left(I S, K_{S}\right)_{n}
$$

is also an isomorphism of degree 1 . Hence we may define

$$
\Gamma:=\omega^{-1} \sigma:\left(K_{S}\right)_{+} \rightarrow K_{S},
$$

a morphism of graded $S$-modules of degree -1 . Since $\sigma$ is injective $\Gamma$ is injective too, proving (i).

Set $a:=-a\left(g r_{A}(I)\right)$. It is clear that $\sigma_{\mid\left(K_{5}\right)_{n}}$ is an isomorphism for any $n<a$, hence

$$
\Gamma_{\mathrm{|}\left(K_{S}\right)_{\mathrm{n}+1}}:\left(K_{S}\right)_{n+1} \rightarrow\left(K_{S}\right)_{n}
$$

is an isomorphism for any $n<a-1$, that is (ii).

To show (iii) take an element $\beta \in\left(K_{S}\right)_{+}$: By definition of the restriction map $\sigma$,

$$
\begin{aligned}
\sigma(\beta): I S & \rightarrow K_{S} \\
s & \rightarrow s \beta .
\end{aligned}
$$

Moreover, by definition of $\pi$

$$
\begin{aligned}
\pi \Gamma(\beta): S_{+} & \rightarrow K_{S} \\
t s & \rightarrow(t s) \Gamma(\beta) .
\end{aligned}
$$

Therefore $s \beta=(t s) \Gamma(\beta)$ for any $s \in I S$. Now assume that $(t s) \alpha=$ $(t s) \Gamma(\beta)$ for any $s \in I S$, where $\alpha \in W_{S}$. Then $(t s)(\alpha-\Gamma(\beta))=0$ for any $s \in I S$ and in particular $(0:(\Gamma(\beta)-\alpha)) \supset S_{+}$, with $h t\left(S_{+}\right)=1$. This implies that $\alpha=\Gamma(\beta)$ since $K_{S}$ is a Cohen-Macaulay $S$-module with $\operatorname{depth}\left(K_{S}\right)=\operatorname{dim}(S)$.

Furthermore for any element $\alpha \in K_{S}$ and any integer $r \geq 1$ we have that $s\left(t^{r} y\right) \alpha=(t s)\left(t^{r-1} y\right) \alpha$ for any $s \in I S$ and $y \in I^{r}$. By (iii) $\Gamma\left(\left(t^{r} y\right) \alpha\right)=\left(t^{r-1} y\right) \alpha$, showing (iv).

Finally it is clear that $\underset{n \geq 2}{\oplus}\left(K_{C}\right)_{n} \simeq\left(\underset{n \geq 2}{\oplus} \underline{\operatorname{Hom}}_{S}\left(I S, K_{S}\right)_{n}\right) / \sigma\left(\left(K_{S}\right)_{+}\right) \simeq$ $\left(K_{S} / \Gamma\left(\left(K_{S}\right)_{+-}\right)\right)(-1)$, hence $(v)$. 
Remark (1.2). If in proposition (1.1) we assume in particular that $a\left(g r_{A}(I)\right) \leq-2$, then $\left(K_{S}\right)_{1} \underset{\sigma}{\simeq} \underline{\operatorname{Hom}}_{S}\left(I S, K_{S}\right)_{1} \underset{\tau}{\simeq} \underline{\operatorname{Hom}}_{S}\left(S_{+}, K_{S}\right)_{0}: \underset{\bar{\psi}}{\simeq}$ $K_{A}$.

Remark (1.3). Next we list some of the cases for which the $a-$ invariant $a\left(g r_{A}(I)\right)$ can be explicitly computed.

(i) Let $I$ be an m-primary ideal in a local ring $(A, \mathfrak{m})$ with infinite residue field. Assume that $g_{A}(I)$ is Cohen-Macaulay. Then

$$
a\left(g r_{A}(I)\right)=\delta(I)-\operatorname{dim}(A)
$$

where $\delta(I)$ is the reduction exponent of $I$, see $[2,(2.4)]$.

(ii) Iet $(A, \mathrm{~m})$ be a Cohen-Macaulay ring and $I$ a strongly CohenMacaulay ideal in $A$ (that is, an idcal such that for any $r \geq 0$ the Koszul homology $H_{r}(K(\underline{a}))$ is zero or a maximal Cohen-Macaulay $A / I$-module, where $\underline{a}$ is any system of generators of $I)$. Assume that the minimal number of generators $\mu\left(I_{p}\right) \leq h t(p)$ for all prime ideals $p \supseteq I$. Then

$$
a\left(g r_{A}(I)\right)=-h t(I),
$$

see $[2,(2.5)]$.

(iii) Let $I$ be an almost complete intersection ideal in a CohenMacaulay ring $(A, \mathrm{~m})$ (we say that $I$ is almost complete intersection if $\mu(I)=h t(I)+1$ and $\mu\left(I_{\mathfrak{p}}\right)=h t(\mathfrak{p})$ for all prime ideals $\left.\mathfrak{p} \in \operatorname{Min}(A / I)\right)$. Then

$$
a\left(g r_{A}(I)\right)=-h t(I)
$$

see $[5,(7.3)]$.

As we have already commented in the introduction, Ikeda proved in [4] that if $\operatorname{grade}(I) \geq 2$ and $R(I)$ is Gorenstein the $a$-invariant $a\left(g r_{A}(I)\right)=$ -2 . This is a particular case of the following result that we may obtain from the proof of proposition (1.1).

Corollary (1.4). Let $(A, \mathrm{~m})$ be a local ring and $I \subset A$ a non principal ideal of $A$ such that $I^{-1}=A$. If $R(I)$ is Gorenstein then $a\left(g r_{A}(I)\right)=$ -2 .

Proof: We use the same notation as in the proof of proposition (1.1). First we show that $a(G) \leq-2$. For this it is enough to sec that $\sigma_{\mid\left(K_{S}\right)}$ is an isomorphism, that is, that any $f \in \operatorname{Hom}_{S}\left(I S, K_{S}\right)_{1}$ is given by the product by an element $a \in\left(K_{S}\right)_{1}$. And this follows immediately from the fact that $\left(K_{S}\right)_{1} \simeq A(S$ is Gorenstein), $I$ generates $I S$ and $\operatorname{Hom}_{A}(I, A)=I^{-1} \doteq A$. Now by proposition (1.1) we have $\left(K_{G}\right)_{-2} \simeq\left(\left(K_{S}\right)_{1} / \Gamma\left(\left(K_{S}\right)_{2}\right)\right) \neq 0$ since $\Gamma$ is injective and $\left(K_{S}\right)_{2} \simeq I$ is not principal. 


\section{Applications}

Throughout this section we shall use the same notation as in the preceding one. Let $I$ be an ideal of $A$ such that the associated graded ring $g r_{A}(I)$ has a canonical module. First we ask when there exists a finitely generated $A$-rnodule $P$ such that $g r_{P}(I):=\underset{n \geq 0}{\oplus} I^{n} P / I^{n+1} P$ is isomorphic to $K_{g r a}(l)(r)$, where $r$ is some integer (see [6] for this question when $I$ is the maximal ideal of $A$ ). Observe that in any case $r=-a\left(g r_{A}(I)\right)$. Suppose that $g r_{A}(I)$ is Cohen-Macaulay (then $A$ is Cohen-Macaulay too). By $[1,(36.1 \mathrm{I})] K_{g r_{A}(l)}$ is a graded $g r_{A}(I)$-module of finite injective dimension with $r k_{k}\left(\operatorname{Ext}_{g r_{A}(l)}^{i}\left(k, K_{g r_{A}(I)}\right)\right)=1$ if $i=\operatorname{dim}\left(g r_{A}(I)\right)$, and 0 otherwise, where $k$ is the residue field of $A$. By standard methods it is easy to show that if $g r_{P}(I) \simeq K_{g r_{A}(I)}(r)$ then $P$ is an $A$-module of finite injective dimension with $r k_{k}\left(\operatorname{Ext}_{A}^{2}(k, P)\right)=1$ if $i=\operatorname{dim}(A)$, and 0 otherwise. Hence $P$ is a canonical module of $A$.

Denote by $(1, t)^{m}$ the $R(I)$-submodule of the polynomial ring $A[t]$ generated by $1, t, \ldots, t^{m}$, where $m \geq 0$, and by $(1, t)^{-1}$ the ideal $I R(I)$. If $P$ is a finitely generated $A$-module let $P(1, t)^{m}$ be the cxtension $P \otimes$ $(1, t)^{n}$. The above question can be answered by means of these modules in the following way:

Theorem (2.1) $([3,(2.4)])$. Let $(A, \mathrm{~m})$ be a local ring and $I \subset A$ be an ideal of $A$ with $h t(I) \geq 1$. Assine that $R(I)$ is Cohen-Macaulay and has a canonical module. Let $a:=-a\left(g r{ }_{A}(I)\right)$. Then the following are equivalent:

(i) $K_{g r_{A}(I)} \simeq g r_{K_{A}}(I)(-a)$.

(ii) $K_{R(I)} \simeq K_{A}(1, t)^{\alpha-2}(-1)$.

Proof: (i) $\Longrightarrow$ (ii) Assume $K_{G} \simeq\left(\underset{n \geq 0}{\oplus} I^{n} K_{A} / I^{n+1} K_{A}\right)(-a)$. First observe that if $a \geq 2$ then $\left(K_{S}\right)_{1} \simeq K_{A}$ by remark (1.2). On the other hand if $a=1$ we have the following diagram with exact sequences

$$
\begin{gathered}
\operatorname{Hom}_{S}\left(S_{+}, K_{S}\right)_{0} \stackrel{\Psi}{\rightarrow} K_{A} \rightarrow 0 \\
\mid \tau \\
0 \rightarrow\left(K_{S}\right)_{1} \underset{\sigma}{\rightarrow} \underline{\operatorname{Hom}}_{S}\left(I S, K_{S}\right)_{1} \underset{\varphi}{\rightarrow}\left(K_{G}\right)_{1} \rightarrow 0
\end{gathered}
$$

where $\tau$ and $\psi$ are isomorphisms. Thus $\left(K_{S}\right)_{1} \simeq \sigma\left(\left(K_{S}\right)_{1}\right)=K$ er $\varphi \simeq$ $\operatorname{Ker}\left(\varphi \tau \psi^{-1}\right)$, and since $\left(K_{C}\right)_{1} \simeq K_{A} / I K_{A}$ and $\varphi \tau \psi^{-1}$ is an epimorphism we have that $\left(K_{S}\right)_{1} \simeq I K_{A}$. 
Claim. If $a \geq 2$ then for any $n \geq a-1$.

$$
\left(K_{S}\right)_{n}=\left(t^{n-a+1} I^{n-a+1}\right)\left(K_{S}\right)_{a-1}
$$

and if $a=1$ then for any $n \geq 1$

$$
\left(K_{S}\right)_{n}=\left(t^{n-a} I^{n-a}\right)\left(K_{S}\right)_{a}
$$

The proof of the claim is by induction on $n$, First assume that $a \geq 2$, and let $n \geq a-1$ such that

$$
\left(K_{S}\right)_{n}=\left(t^{n-a+1} I^{n-a+1}\right)\left(K_{S}\right)_{a-1}
$$

Consider the diagram we used in the proof of proposition (1.1)

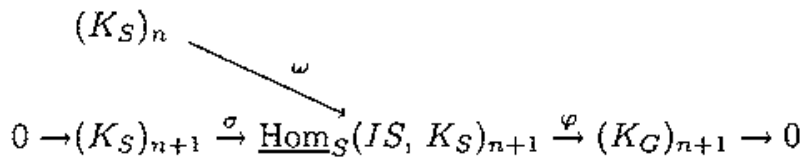

By definition

$$
\Gamma\left(\left(K_{S}\right)_{n+1}\right)=\omega^{-1} \sigma\left(\left(K_{S}\right)_{n+1}\right)=\omega^{-1}(\operatorname{Ker}(\varphi))=\operatorname{Ker}(\omega \varphi),
$$

while by assumption $\left(K_{G}\right)_{n+1}=I^{n+1-a} K_{A} / I^{n+2-a} K_{A}$ with $K_{A} \simeq\left(K_{S}\right)_{a-1}$. Hence $\left(K_{G}\right)_{n+1} \simeq I^{n+1-a}\left(K_{S}\right)_{a-1} / I^{n+2-a}\left(K_{S}\right)_{a-1}=$ $\left(K_{S}\right)_{n} / I\left(K_{S}\right)_{n}$, and since $\omega \varphi$ is an cpimorphism this implies that $K \operatorname{Ker}(\omega \varphi)=I\left(K_{S}\right)_{n}$. Therefore $\Gamma\left(\left(K_{S}\right)_{n+1}\right)=I\left(K_{S}\right)_{n}=\Gamma\left((t I)\left(K_{S}\right)_{n}\right)$ by proposition (1.1), (iv) and since $\Gamma$ is injective we get that

$$
\left(K_{S}\right)_{n+1}=(t I)\left(K_{S}\right)_{n}=\left(t^{n-2+2} I^{n-a+2}\right)\left(K_{S}\right)_{a-1} \text {. }
$$

With similar arguments we can also proof the clam when $a=1$.

Therefore we can write

$$
\begin{aligned}
K_{S} & =\left(K_{S}\right)_{1} \oplus \cdots \oplus\left(K_{S}\right)_{a-1} \oplus(t I)\left(K_{S}\right)_{a-1} \\
& \oplus\left(t^{2} I^{2}\right)\left(K_{S}\right)_{a-1} \oplus \cdots
\end{aligned}
$$

with $\left(K_{S}\right)_{1} \underset{a}{a} K_{A}$ if $a \geq 2$, and

$$
K_{S}=\left(K_{S}\right)_{1} \oplus(t I)\left(K_{S}\right)_{1} \oplus\left(t^{2} t^{2}\right)\left(K_{S}\right)_{1} \oplus \cdots
$$


with $\left(K_{S}\right)_{1} \underset{h}{ } \widetilde{L} K_{A}$ if $a=1$.

Suppose $a \geq 2$. By definition

$K_{A}(1, t)^{a-2}=K_{A} \oplus t K_{A} \oplus \cdots \oplus t^{n-2} K_{A} \oplus t^{a-1} I K_{A} \oplus t^{a} J^{2} K_{A} \oplus \cdots$

Consider the map

$$
\xi: K_{S} \rightarrow K_{A}(1, t)^{\alpha-2}
$$

defined by $\xi_{\mid\left(K_{s}\right),}=g$ and $\xi_{\mid\left(K_{S}\right)_{n}}=t\left(\xi_{\mid\left(K_{s}\right)_{n-1}} \Gamma\right)$ if $n \geq 2$. $\xi$ is a morphism of graded $S$-modules, for if $\alpha \in\left(K_{S}\right)_{n}$ and $a \in I^{r}, \xi\left(\left(t^{r} a\right) \alpha\right)=$ $t \xi\left(\Gamma\left(\left(t^{r} a\right) \alpha\right)\right)=t \xi\left(\left(t^{r-1} a\right) \alpha\right)$ by proposition (1.1). (iv) and applying this repeatdly we get $\xi\left(\left(t^{r} a\right) \alpha\right)=t^{r} \xi(a \alpha)=\left(t^{r} a\right) \xi(\alpha)$.

It is clear that $\xi$ is injective ( $\Gamma$ it is), hence to show that $\xi$ is an isomorphism we must see that $\xi\left(\left(K_{S}\right)_{n}\right)=\left(K_{A}(1, t)^{a-2}\right)_{n-1}$ for any $n$. This is trivial for $n<a$, thus assume $n \geq a$. By the claim $\left(K_{S}\right)_{n}=$ $(t I)\left(K_{S}\right)_{n-1}$, hence $\Gamma\left(\left(K_{S}\right)_{n}\right)=I\left(K_{S}\right)_{n-1}$ by proposition (1.1), (iv). By induction we then get that $\xi$ is an isomorphism. Since it is of degree -1 we finally obtain

$$
K_{S} \simeq K_{A}(1, t)^{n-2}(-1)
$$

Similarly we could proof the case $a=1$.

(ii) $\Longrightarrow$ (i) If $a \geq 2$ this is a direct consequence of proposition (1.1), (v). Hence assume $a=1$ and $K_{S} \simeq K_{A}(1, t)^{-1}(-1)$. Consider the exact. sequence

$$
0 \rightarrow K_{S} \stackrel{o}{\rightarrow} \underline{\operatorname{Hom}}_{S}\left(I S, K_{S}\right) \stackrel{\varphi}{\rightarrow} K_{C} \rightarrow 0
$$

Passing to the completion if necessary and by local duality $[1,(36.8)]$ we obtain the exact sequence

$$
0 \rightarrow K_{A} I S(-1) \rightarrow K_{A} S(-1) \rightarrow K_{G} \rightarrow 0
$$

thus $K_{C} \simeq g r_{K_{A}}(I)(-1)$ as we wanted to show.

Now we can also obtain the following version of Ikeda's result characterizing when the Recs algebra is Gorenstein:

Theorem (2.2) $([4,(3.1)])$. Let $(A, \mathrm{~m})$ be a local ring and $I$ be an ideal of $A$. Sinpose that $R(I)$ is Cohen-Macaulay and $I^{-1}=A$. The following conditions are equivalent:

(i) $R(I)$ is Corcnstein.

(ii) $K_{A} \simeq A$ and $K_{g r r_{A}(I)} \simeq g r_{A}(I)(-2)$. 
Proof: (i) $\Longrightarrow$ (ii) By corollary (1.4) $a(G)=-2$, and by remark (1.2) $K_{A} \simeq A$. Hence by theorem (2.1) $K_{G} \simeq G(-2)$.

(ii) $\Longrightarrow$ (i) By theorem $(2.1)$ we get $K_{S} \simeq(1, t)(-1)=S(-1)$, and $S$ is Gorenstein since by hypothesis $S$ is Cohen-Macaulay.

Remark (2.3). Assume under the hypothesis of theorem (2.1) that $g r_{A}(I)$ is Gorenstein and $a\left(g r_{A}(I)\right)=-1$. Then $K_{R(I)} \simeq I S(-1)$, thus in particular $I \simeq\left(K_{R(I)}\right)_{1}$ and $R(I)$ cannot bc Gorenstein if $I$ is not principal.

\section{Acknowledgements.}

I must thank M. Herrmann, J. Ribbe and H. Hiri for the discussions we had about this problem during my stay in the Mathematical Institute of the University of Cologne (Germany). I was partially supported by DGICYT-grant PB 88-0224 (Spain).

\section{References}

1. M. Herrmann, S. IKeda and U. Orbanz, "Equimultiplicity and Blowing Up," Springer-Verlag, Berlin Heidelberg, 1988.

2. M. Herrmann, J. Ribbe and S. Zarzuela, On the Gorenstein property of Rees and form rings of powers of ideals, to appear in Transactions of the American Mathematical Society.

3. J. Herzog, A. Simis and W. V. Vasconcelos, On the canonical module of the Rees algebra and the associated graded ring of an ideal, Journal of Algebra 105 (1987), 285-302.

4. S. IKEDA, On the Gorensteinness of Rees algebras over local rings, Nagoya Mathematical Journal 102 (1986), 135-154.

5. J. RlBBe, Dissertation, Universität zu Köln (1991).

6. A. OoIsHI, On the associated graded modules of canonical modules; Journal of Algebra 141, no. 1 (1991), 143-157.

Departament d'Àlgebra i Geonctria

Facultat de Matematiques

Universitat de Barcelona

Gran Via 585

E-08007 Barcclona

SPAIN

Rebut el 18 de Desembre de 1991 